\title{
A rare cause of diarrhea: pancreatic VIPoma
}

A 45-year-old man presented with watery, large-volume diarrhea for 4 years and $13.6 \mathrm{~kg}$ (30 lb) weight loss. Multiple admissions and investigations for dehydration, including computed tomography (CT), esophagogastroduodenoscopy and colonoscopy with biopsies, and capsule endoscopy, yielded negative findings. Laboratory workup, including complete blood count, tissue transglutaminase, thyroid-stimulating hormone, cortisol, serum/urine protein electrophoresis, fecal fat, calprotectin, and erythrocyte sedimentation rate (ESR)/C-reactive protein (CRP), was normal [1]. Workup for infectious diseases was negative for parasites, Clostridium difficile, and human immunodeficiency virus (HIV). Stool electrolytes were consistent with secretory diarrhea based on an osmolar gap of $40 \mathrm{mOsm} / \mathrm{kg}$. Neurohormonal testing revealed elevated chromogranin A $(320 \mathrm{ng} / \mathrm{mL}$, normal range: 0-95) and vasointestinal peptide (VIP) (360 pg/mL, normal range: 0-60), suspicious for neuroendocrine tumor producing VIP (VIPoma). Prior CT scans were inconclusive for neuroendocrine tumor, but octreotide scan showed marked accumulation of tracer in the pancreas and liver ( $\mathbf{F i g . 1} \mathbf{a}, \mathbf{b}$ ). Endoscopic ultrasound (EUS) revealed a hypoechoic mass measuring $25 \times 30 \mathrm{~mm}$ in the pancreatic neck ( $\bullet$ Fig. $1 \mathrm{c}$ ) and a hypoechoic $7 \times 8 \mathrm{~mm}$ lesion in the right hepatic lobe. Fine needle aspiration showed mononuclear cells with granular cytoplasm and stippled chromatin, hyperchromatic nuclei, and stained positive for synaptophy$\sin (\bullet$ Fig. 2) consistent with a neuroendocrine tumor. The patient had symptomatic improvement with administration of octreotide, and the diagnosis of VIPoma was confirmed.

VIPoma is rare, with worldwide incidence of 1 in 10000000 [2], and $80 \%$ have metastasized before diagnosis [3]. Described as "pancreatic cholera," symptoms include 3-5L/day of watery diarrhea, hypokalemia, achlorhydria, and acidosis. Diagnosis is established via octreotide scan (sensitivity $58 \%-86 \%$ ) and EUS (sensitivity $87 \%$ ) [4]. Complete resection is curative but rarely possible due to the extent of disease at diagnosis [2,3]. Symptomatic palliation may be achieved with debulking/chemoembolization and/or administration of octreotide [2,3].

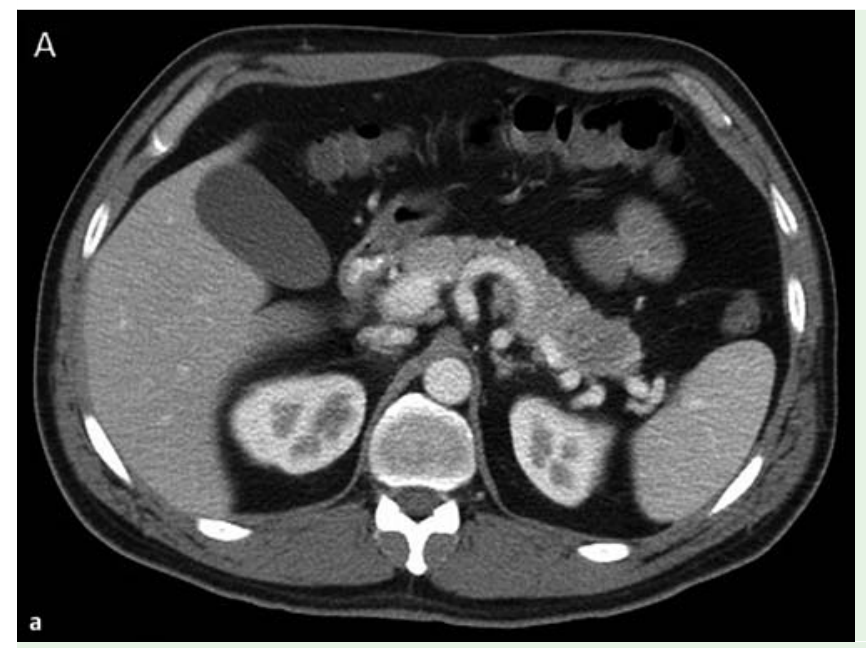

Fig. 1 a, b Computed tomography (CT) and octreotide scanning findings in pancreas suggestive of neuroendocrine tumor in a 45-year-old man presenting with watery, large-volume diarrhea for 4 years and massive weight loss. c Endosonographic findings of tumor in pancreatic neck.
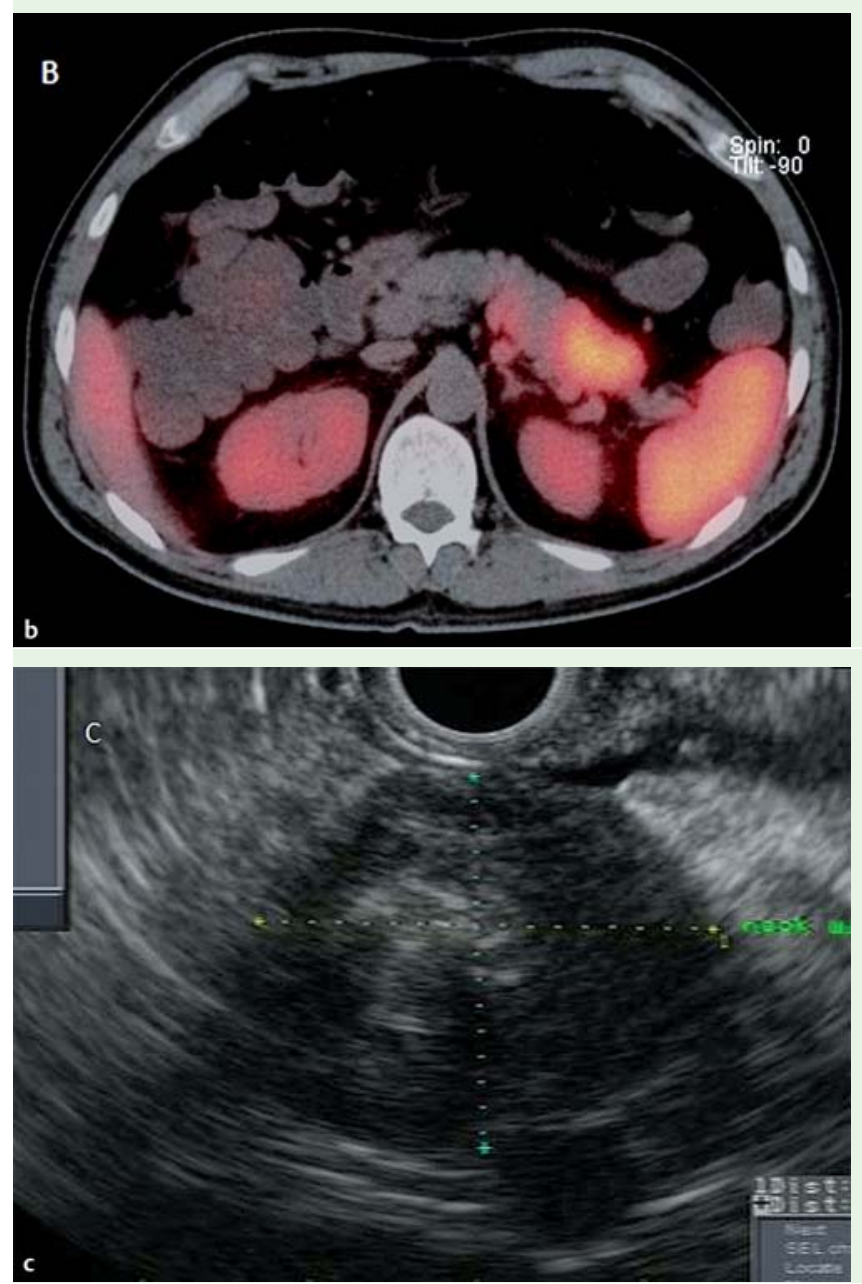

Pancreatic neuroendocrine tumors are rare and can be difficult to diagnose. They should be suspected in those with longstanding secretory diarrhea. EUS has an increasingly recognized role in diagnosis and staging $[3,4]$.
Endoscopy_UCTN_Code_CCL_1AF_2AZ_3AB

Competing interests: N. J. Samadder is a speaker for Cook Medical. 


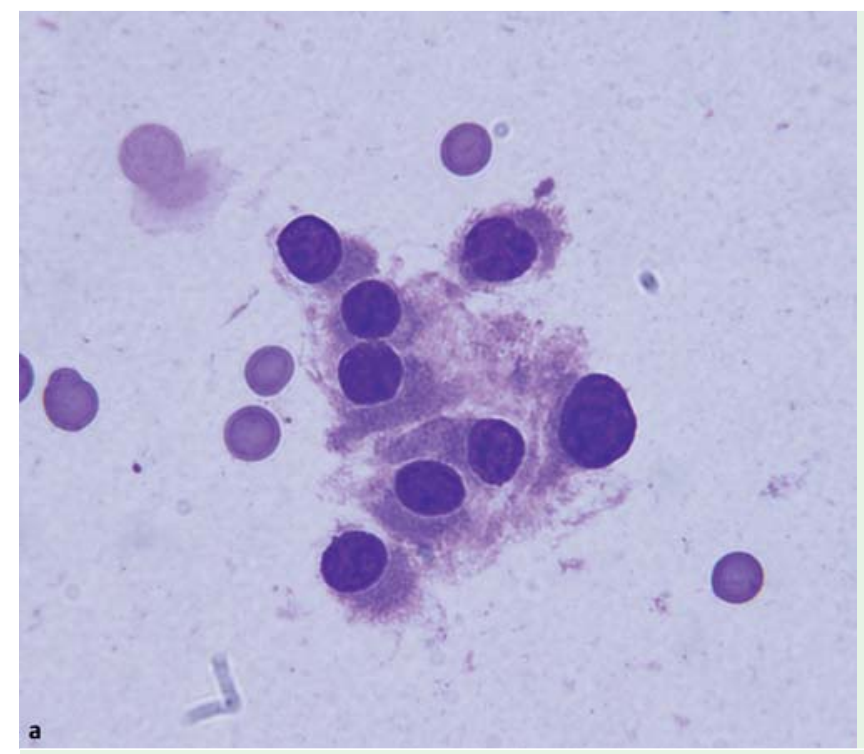

Fig. 2 Histological features of neuroendocrine tumor. a Mononuclear cells with granular cytoplasm and stippled chromatin. b Hyperchromatic nuclei. c Positive synaptophysin staining.

\section{J. B. Johnson ${ }^{1}$, L. Marsden ${ }^{2}$, N. J. Samadder ${ }^{1}$}

${ }^{1}$ Department of Medicine (Gastroenterology), Huntsman Cancer Institute and University of Utah, Salt Lake City, Utah, United States of America

${ }^{2}$ Department of Pathology, Huntsman Cancer Institute and University of Utah, Salt Lake City, Utah, United States of America

\section{References}

1 Fine KD, Schiller LR. AGA technical review on the evaluation and management of chronic diarrhea. Gastroenterology 1999; 116: $1464-1486$

2 Song S, Shi R, Li Bi et al. Diagnosis and treatment of pancreatic vasoactive intestinal peptide endocrine tumors. Pancreas 2009; 38: $811-814$

3 Batcher E, Madaj P, Gianoukakis AG. Pancreatic neuroendocrine tumors. Endocr Res 2011; 36: 35-43

$4 \mathrm{Kim}$ MK. Endoscopic ultrasound in gastroenteropancreatic neuroendocrine tumors. Gut Liver 2012; 6: 405-410

\section{Bibliography}

DOI http://dx.doi.org/

10.1055/s-0033-1344411

Endoscopy 2013; 45: E311-E312

(c) Georg Thieme Verlag KG

Stuttgart · New York

ISSN 0013-726X

\section{Corresponding author}

\section{N. J. Samadder}

Huntsman Cancer Institute

2000 Circle of Hope

Salt Lake City

UT 84112

USA

Fax: +801-581-7476

jewel.samadder@hsc.utah.edu 